\title{
Experimental Study of the Surface Wave around Hydrofoils
}

\author{
C. Kang*, M. G. Yang
}

School of Energy and Power Engineering, Jiangsu University, Zhenjiang 212013, China

Email: kangcan@ujs.edu.cn

\begin{abstract}
The evolution of surface wave presents unsteady and transient characteristics. In many engineering systems involving hydraulic machinery, rotary propeller, or hydraulic constructions etc, surface wave can be critical under certain operating conditions. Recent years' researches have brought many helpful and instructive achievements both in academic and application levels. And the numerical simulation work has been mainly focused. However, numerical simulation is restricted in flows near the simple boundaries and numerical simulation has to be testified and validated by corresponding experimental results. So an accurately observed wave profile and its evolution in experiment can be very useful both for analytical and numerical work. From the viewpoint of experiment, common photography can hardly capture the instantaneous variation of the wave surface. To study the surface wave profile and surface wave's evolution around different hydrofoils, an experimental rig was built based on a water tunnel platform. Different hydrofoils were adopted respectively in transparent experimental part of the flow system. High speed photography was applied in the experiment to record the instant variation of the wave's profile. Pressure transducers and a computer-aided data collection system were used to measure the pressures in different points.

According to recorded wave front profiles, the affection of different hydrofoils and different attack angles on wave front's evolution were analyzed. The results show that:(1) under the action of boundary layer, two separated wave filaments appear when the surface wave deviates from the hydrofoil's surface; (2) the travel velocity of the wave separating from the leading edge is obviously larger that that of the wave separating from the trailing edge; (3) the consequence of wave-wave action is the occurrence of wake vortex element which remains it's shape for a relative long period.
\end{abstract}

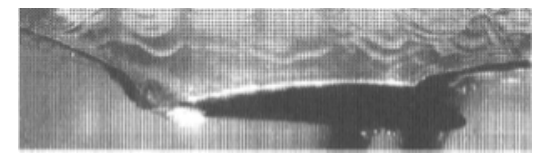

Figure 1:Wave profile at $\mathrm{T}=100 \mathrm{~ms}$

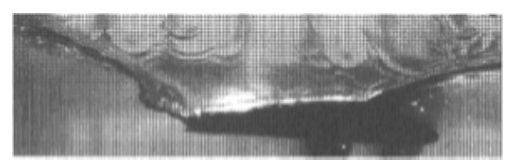

Figure 2: Wave profile at $\mathrm{T}=230 \mathrm{~ms}$

\section{REFERENCES}

1. Gu H B, Li Y B, Li S W, Zhang Q H. The level set and particle level set method for tracing interface. Journal of Hydrodynamics (A), 2005; 20(2): 152-160

2. Liu F, Hu F. Application of lattice Boltzmann method in simulating reflection and diffraction of surge waves. Journal of Hydraulic Engineering, 2004; (3): 22-28

3. Li H X, Yang D, Chen T K, et al. A formulation for the level set method and its applications in gas-liquid two-phase flow simulations. Journal of Engineering Thermophysics. 2001; 22(2): 233-236

4. Gao Y L, Liu Q H. Application of level set method to numerical simulating free surface flow. Yantai Normal University Journal(Natural Science), 2005; 21(1): 14-16 